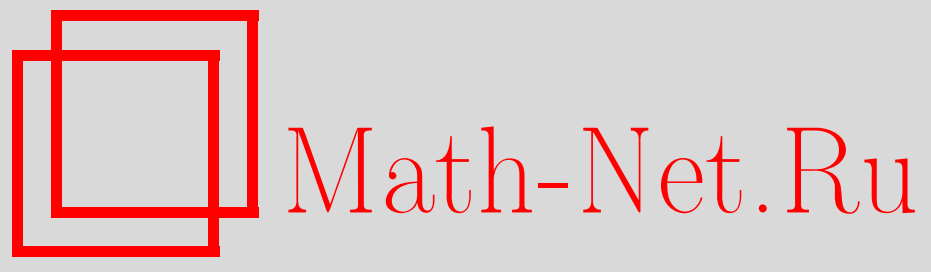

Н. И. Жукова, Аттракторы слоений с трансверсальной параболической геометрией ранга один, Матем. заметки, 2013, том 93, выпуск 6, 944-946

DOI: https://doi.org/10.4213/mzm10249

Использование Общероссийского математического портала Math-Net.Ru подразумевает, что вы прочитали и согласны с пользовательским соглашением http://www . mathnet.ru/rus/agreement

Параметры загрузки:

IP: 107.22 .136 .117

26 апреля 2023 г., 04:28:09

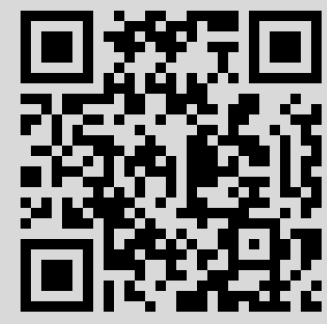




\section{Аттракторы слоений с трансверсальной параболической геометрией ранга один}

\section{Н. И. Жукова}

Цель работы - представить единый метод исследования слоений с трансверсальной параболической геометрией ранга один. Мы развиваем технику Франса [1] и идеи, примененные нами к конформным слоениям [2].

1. Параболические геометрии ранга один. Пусть $G$ - простая группа Ли действительного ранга один с конечным центром, а $P$ - ее параболическая подгруппа. Сохраним обозначения из [1]. Пусть $\mathbf{X}=G / P$. Такие однородные пространства $\mathbf{X}$ являются границами $\mathbf{X}=\partial \mathbf{H}_{\mathbb{K}}^{d}$ различных $d$-мерных гиперболических пространств $\mathbf{H}_{\mathbb{K}}^{d}$. Здесь $\mathbb{K}$ есть $\mathbb{R}, \mathbb{C}$, $\mathbb{H}$ или алгебра октанионов $\mathbb{O}$. Предполагается, что $d \geqslant 2$, если $\mathbb{K}=\mathbb{R}$, и $d=2$ для $\mathbb{K}=\mathbb{O}$, во всех остальных случаях $d \geqslant 1$.

Таким образом, $\mathbf{X}=G / P=\partial \mathbf{H}_{\mathbb{K}}^{d}$, где $G=\operatorname{Iso}\left(\mathbf{H}_{\mathbb{K}}^{d}\right)$ и $P$ - стационарная подгруппа группы $G$ в некоторой точке границы $\partial \mathbf{H}_{\mathbb{K}}^{d}$. Известен полный список этих групп и соответствующих однородных пространств:

- $G=S O(1, d), d \geqslant 2$ при $\mathbb{K}=\mathbb{R}$, причем $\mathbf{X}=\partial \mathbf{H}_{\mathbb{R}}^{d}$ есть сфера $\mathbf{S}^{d-1}$;

- $G=S U(1, d), d \geqslant 1$ при $\mathbb{K}=\mathbb{C}$, причем $\mathbf{X}=\partial \mathbf{H}_{\mathbb{C}}^{d}$ есть сфера $\mathbf{S}^{2 d-1}$;

- $G=\operatorname{Sp}(1, d), d \geqslant 1$ при $\mathbb{K}=\mathbb{H}$, причем $\mathbf{X}=\partial \mathbf{H}_{\mathbb{H}}^{d}$ есть сфера $\mathbf{S}^{4 d-1}$;

- $G=F_{4}^{-20}$ при $\mathbb{K}=\mathbb{O}$, причем $\mathbf{X}=\partial \mathbf{H}_{\mathbb{K}}^{d}$ есть сфера $\mathbf{S}^{15}$.

Определения и факты о картановых и параболических геометриях можно найти, например, в книге Чапа и Словака [3]. Пусть $G$ - группа Ли и $P$ - ее замкнутая подгруппа, а $\mathfrak{g}$ и $\mathfrak{p}$ - их алгебры Ли. Пусть $B(N, P)-P$-расслоение с проекцией $p: B \rightarrow N$. Невырожденная $P$-эквивариантная $\mathfrak{g}$-значная 1-форма $\omega$ на $B$ называется картановой связностъю. Пара $\xi=(B(N, P), \omega)$ называется картановой геометрией типа $(G, P)$, а $(N, \xi)$ - картановым многообразием.

Картанова геометрия $\xi=(B(N, P), \omega)$ типа $(G, P)$, где $G$ - простая группа Ли действительного ранга один с конечным центром, а $P$ - ее параболическая подгруппа, называется параболической геометрией ранга один или, для краткости, - $P_{1}$-геометрией. Параболическая геометрия называется регулярной, если ее кривизна согласована с градуировкой алгебры Ли g в смысле [1]. Картанова геометрия $\left(G(\mathbf{X}, P), \omega_{G}\right)$, где $\omega_{G}$ - форма Маурера-Картана на $G$, обозначается через $\xi^{0}$.

Пусть $\xi=(B(N, P), \omega)$ и $\xi^{\prime}=\left(B^{\prime}\left(N^{\prime}, P\right), \omega^{\prime}\right)$ - картановы геометрии одного типа $(G, P)$. Изоморфизмом $\xi$ и $\xi^{\prime}$ называется такой диффеоморфизм $\widehat{f}: B \rightarrow B^{\prime}$, что $f^{*} \omega^{\prime}=\omega$ и $\widehat{f} \circ R_{a}=R_{a} \circ \widehat{f}$ для всех $a \in P$, где $R_{a}$ - правое действие элемента $a \in P$ на $B$ и $B^{\prime}$ соответственно. Проекция $f: N \rightarrow N^{\prime}$ диффеоморфизма $\widehat{f}$ называется изоморфизмом картановых многообразий $(N, \xi)$ и $\left(N^{\prime}, \xi^{\prime}\right)$. Обозначим через $\operatorname{Aut}(N, \xi)$ группу автоморфизмов $(N, \xi)$.

Везде далее $\xi=(B(N, P), \omega)$ - регулярная параболическая геометрия ранга один.

2. Существенность локального изоморфизма. Пусть $\gamma: U \rightarrow V$ - такой локальный изоморфизм параболического многообразия $(N, \xi)$, что $\gamma(v)=v \in U \cap V$, и $\widehat{\gamma}-$ (какойлибо) локальный изоморфизм геометрии $\xi$ с проекцией $\gamma$. Зафиксируем точку $\widehat{v} \in p^{-1}(v)$. Существует единственный элемент $a$ из группы $P$, удовлетворяющий равенству $\widehat{\gamma}(\widehat{v})=\widehat{v} a$. Рассмотрим индуцированную подгруппу $\Gamma_{\widehat{v}}:=\langle a\rangle$ группы $P$, порожденную элементом $a$. Нетрудно проверить корректность следующего определения.

Работа выполнена при поддержке Министерства образования и науки РФ "Научные и научнопедагогические кадры" (проект № 14.В37.21.0361) и проект № 1.1907.2011.

DOI: $10.4213 / \mathrm{mzm} 10249$ 
ОПРедЕлЕниЕ 1. Если локальный изоморфизм $\gamma$ геометрии $\xi$ имеет такую неподвижную точку $v$, что индуцированная группа $\Gamma_{\widehat{v}}$, где $p(\widehat{v})=v$, не является относительно компактной подгруппой группы Ли $P$, то $\gamma$ называется существенным (в точке $v$ ). В противном случае локальный изоморфизм $\gamma$ называется несущественным.

Применяя отображение развертки для параболической геометрии $\xi$, мы показываем, что техника, использованная Франсом в [1] для группы автоморфизмов $\operatorname{Aut}(N, \xi)$, применима к псевдогруппе локальных изоморфизмов $\mathcal{H}(N, \xi)$ геометрии $(N, \xi)$. Благодаря этому мы получили следующее достаточное локальное условие для выполнения гипотезы Лихнеровича для произвольной параболической геометрии ранга один.

ТЕОрема 1. Пусть $\xi$ - произвольная регулярная параболическая геометрия ранга один на $N$. Если существует существенный локальный изоморфизм параболического многообразия $(N, \xi)$, то $(N, \xi)$ изоморфно либо параболическому многообразию $\left(\mathbf{X}, \xi^{0}\right)$, если $N$ компактно, либо параболическому многообразию $\left(\mathbf{X} \backslash\{\nu\}, \xi^{0} \mid \mathbf{X} \backslash\{\nu\}\right)$, где $\nu \in \mathbf{X}$, в противном случае.

Нами доказан следующий критерий собственности действия группы автоморфизмов $\operatorname{Aut}(N, \xi)$ параболического многообразия $(N, \xi)$.

Теорема 2. Полная группа автоморфизмов $\operatorname{Aut}(N, \xi)$ действует собственно на $N$ тогда и только тогда, когда она не содержит существенных автоморфизмов.

Из теорем 1 и 2 вытекает основная теорема Франса из [1], согласно которой если группа автоморфизмов регулярной параболической геометрии ранга один $(N, \xi)$ действует на $N$ несобственно, то выполняется утверждение теоремы 1.

Другой критерий собственности действия на $N$ группы автоморфизмов $P_{1}$-геометрии $\xi$ в терминах вейлевых структур получен Альтом в [4].

3. Критерий римановости $P_{1}$-слоения. Слоение $(M, F)$, заданное $N$-коциклом $\left\{U_{i}\right.$, $\left.f_{i},\left\{\gamma_{i j}\right\}\right\}_{i, j \in J}$, имеет трансверсалъную геометрию $\xi$ и называется $P_{1}$-слоением, если на $N$ задана такая регулярная параболическая геометрия ранга один $\xi$, что каждый $\gamma_{i j}-$ локальный изоморфизм $(N, \xi)$.

Напомним, что псевдогруппа локальных изоморфизмов, порожденная локальными изоморфизмами $\gamma_{i j}, i, j \in J$, называется псевдогруппой голономии слоения $(M, F)$ и обозначается $\mathcal{H}(M, F)$. Пусть $L-$ произвольный слой слоения $(M, F)$, возьмем $x \in L \cap U_{i}, i \in J$. Группа ростков в точке $v=f_{i}(x)$ локальных диффеоморфизмов из $\mathcal{H}(M, F)$ называется (ростковой) группой голономии слоя $L$. Имеет смысл следующее определение.

ОПРедЕлЕниЕ 2. Группа голономии слоя $L$ называется существенной, если она содержит росток существенного локального изоморфизма в точке $v=f_{i}(x)$, где $x \in L \cap U_{i}$.

Применяя предложение 2, доказанное автором в [2], мы получили следующий критерий римановости исследуемых слоений.

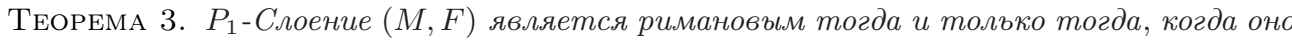
не имеет слоев с существенной группой голономии.

4. Существования аттракторов. Напомним, что любое объединение слоев слоения $(M, F)$ называется насыщенным подмножеством $M$.

ОПределЕниЕ 3. Непустое замкнутое насыщенное подмножество $\mathcal{M}$ в $M$ называется аттрактором слоения $(M, F)$, если существует такая насыщенная открытая окрестность $\operatorname{Attr}(\mathcal{M})$ множества $\mathcal{M}$, что замыкание каждого слоя из $\operatorname{Attr}(\mathcal{M}) \backslash \mathcal{M}$ содержит $\mathcal{M}$. При этом $\operatorname{Attr}(\mathcal{M})$ называется бассейном аттрактора $\mathcal{M}$. 
Минимальное множество слоения $(M, F)$ есть непустое замкнутое насыщенное подмножество $M$, не имеющее собственных подмножеств, обладающих этими свойствами. В отличие от компактных многообразий, на некомпактных многообразиях существуют слоения без минимальных множеств. Применение теорем 1 и 3 позволило нам доказать следующую теорему без предположения компактности слоеного многообразия.

Теорема 4. Для любого $P_{1}$-слоения $(M, F)$ выполняется одно из двух следующих утверждений:

(1) слоение $(M, F)$ риманово;

(2) существует слой с существенной группой голономии; замыкание $\mathcal{M}=\bar{L}$ любого слоя L с существенной группой голономии является аттрактором и минимальным множеством, причем сужение слоения на бассейн $\operatorname{Attr}(\mathcal{M})$ aттрактора $\mathcal{M}$ есть $(G, \mathbf{X})$-слоение; объединение $\mathcal{K}$ замыканий всех слоев с существенной группой голономии образует замкнутое подмножество $M$, а ограничение $\left(M_{0}, F_{M_{0}}\right)$ этого слоения на открытое подмножество $M_{0}:=M \backslash \mathcal{K}$ - риманово слоение.

В случае, когда $(M, F)$ - конформное слоение коразмерности $q \geqslant 3$, подобный результат был получен автором в [2].

5. Строение $P_{1}$-слоений на компактных многообразиях. Используя теорему 4 и рассуждения автора для конформных слоений [2], доказана

Теорема 5. Если $(M, F)$ - $P_{1}$-слоение на компактном многообразии $M$, то выполняется одно из следующих двух утверждений:

(1) слоение $(M, F)$ полное риманово;

(2) слоение $(M, F)$ трансверсально однородное, имеет непустое конечное множество минимальных множеств $\mathcal{M}_{j}, j=1, \ldots, m$, являющихся аттракторами, причем каждый слой принадлежит бассейну по крайней мере одного из этих аттракторов.

Теорему 5 можно рассматривать как положительный ответ на аналог гипотезы Лихнеровича для $P_{1}$-слоений.

Для конформных слоений коразмерности $q \geqslant 3$ этот результат получен нами в [2], а трансверсальная однородность доказана Таркини [5] при дополнительном предположении трансверсальной аналитичности конформного слоения.

Следствие. Пусть $(M, F)$ - собственное нериманово $P_{1}$-слоение на компактном многообразии $M$. Тогда $(M, F)-(G, \mathbf{X})$-слоение, имеющее непустое конечное множество компактных слоев $L_{j}, j=1, \ldots, m \geqslant 1$, причем каждый $L_{j}$ - аттрактор и любой слой этого слоения принадлежит бассейну по крайней мере одного из этих аттракторов.

Примеры конформных слоений с различные минимальными множествами-аттракторами построены нами в [6].

\section{СПИСОК ЦИТИРОВАННОЙ ЛИТЕРАТУРЫ}

[1] C. Frances, Ann. Sci. École Norm. Sup. (4), 40:5 (2007), 741-764. [2] Н. И. Жукова, Сиб. матем. журн., 52:3 (2011), 555-574. [3] A. Čap, J. Slovák, Parabolic Geometries. I. Background and General Theory, Math. Surveys Monogr., 154, Amer. Math. Soc., Providence, RI, 2009. [4] J. Alt, SIGMA, 7 (2011), 039, 16 pp. [5] C. Tarquini, Ann. Inst. Fourier (Grenoble), 52:2 (2004), 453-480. [6] Н. И. Жукова, Матем. сб., 203:3 (2012), 79-106.

\section{Н. И. Жукова}

Поступило

Нижегородский государственный 